\title{
Antigenic Drift of the Hemagglutinin from an Influenza A (H1N1) pdm09 Clinical Isolate Increases its Pathogenicity In Vitro
}

\author{
Lei Xing ${ }^{1,3} \cdot$ Yunbo Chen ${ }^{2}$ - Boqian Chen ${ }^{2} \cdot$ Ling Bu ${ }^{1,3} \cdot$ Ying Liu ${ }^{4} \cdot$ Zhiqi Zeng $^{1} \cdot$ Wenda Guan $^{1} \cdot$

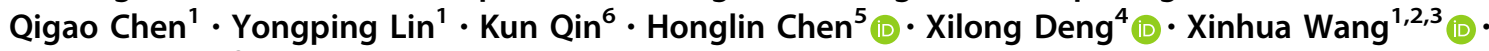 \\ Wenjun Song ${ }^{1,3,5}$
}

Received: 6 January 2021 / Accepted: 12 April 2021 / Published online: 9 June 2021

(c) Wuhan Institute of Virology, CAS 2021

\begin{abstract}
The influenza A (H1N1) pdm09 virus emerged in 2009 and has been continuously circulating in humans for over ten years. Here, we analyzed a clinical influenza A (H1N1) pdm09-infected patient case hospitalized for two months in Guangdong (from December 14, 2019 to February 15, 2020). This isolate, named A/Guangdong/LCF/2019 (LCF/19), was genetically sequenced, rescued by reverse genetics, and phylogenetically analyzed in the context of other relevant pdm09 isolates. Compared with earlier isolates, this pdm09 virus's genetic sequence contains four substitutions, S186P, T188I, D190A, and Q192E, of the hemagglutinin (HA) segment at position 186-192 (H3 numbering) in the epitope Sb, and two of which are located at the 190-helix. Phylogenetic analysis indicated that the epitope Sb started undergoing a rapid antigenic change in 2018. To characterize the pathogenicity of this novel substitution motif, a panel of reassortant viruses containing the LCF/ 2019 HA segment or the chimeric HA segment with the four substitutions were rescued. Kinetic growth data revealed that the reassortant viruses, including the LCF/2019 with the PTIAAQE substitution, propagated faster than those rescued ones having the STTADQQ motif in the epitope Sb in Madin-Darby Canine Kidney (MDCK) cells. The HI test showed that the binding activity of escape mutant to 2018 pdm09 sera was weaker than GLW/2018, suggesting that old vaccines might not effectively protect people from infection. Due to the difference in the selection of vaccine strains, people vaccinated in the southern hemisphere could still suffer a severe infection if infected with this antigenic drift pdm09 virus.
\end{abstract}

Keywords HA antigenic drift - Influenza A (H1N1) pdm09 virus · HA epitope Sb

Lei Xing and Yunbo Chen have contributed equally to this work.

Wenjun Song

wjsong@hku.hk

Xinhua Wang

xinhuaw@gzhmu.edu.cn

Xilong Deng

xlodeng@126.com

$\square$ Honglin Chen

hlchen@hku.hk

$1 \quad$ State Key Laboratory of Respiratory Disease, National Clinical Research Center for Respiratory Disease, Guangzhou Institute of Respiratory Health, The First Affiliated Hospital of Guangzhou Medical University, Guangzhou 510180, China

2 Artemisinin Research Center, Guangzhou University of Chinese Medicine, Guangzhou 510405, China
3 Institute of Integration of Traditional and Western Medicine, The First Affiliated Hospital of Guangzhou Medical University, Guangzhou 510180, China

4 Intensive Care Unit, Guangzhou No.8 People's Hospital of Guangzhou Medical University, Guangzhou 510060, China

5 State Key Laboratory of Emerging Infectious Diseases, Department of Microbiology, The University of Hong Kong, Hong Kong SAR, China

6 China CDC, National Institute for Viral Disease Control and Prevention, Beijing 100052, China 


\section{Introduction}

Influenza A viruses are classified into subtypes based on two surface proteins: hemagglutinin (HA) and neuraminidase (NA). There are $18 \mathrm{HA}$ subtypes and $11 \mathrm{NA}$ subtypes (H1-H18 and N1-N11, respectively), expressing various subtype combinations and circulating in nature (Latorre-Margalef et al. 2014; Song et al. 2014; Wu et al. 2014). In April 2009, a novel swine-origin influenza A H1N1 (S-OIV) virus, classified as influenza A (H1N1) pdm09 or pdmH1N1, first emerged in Mexico and quickly replaced the traditional seasonal H1N1 to become the dominant H1N1 subtype (Novel Swine-Origin Influenza Novel Swine-Origin Influenza AVIT et al. 2009). To date, the influenza A (H1N1) pdm09 virus has been circulating in the world for more than one decade and has become a global threat to public health.

Vaccination reduces the risk of flu illness when most circulating flu viruses are well-matched to the vaccine. However, antigenic drift, which continually happens over time as the virus replicates, might decrease the vaccine's effectiveness. Most flu shots are designed to target HA surface proteins/antigens of influenza virus. Like other subtypes, pdm09 HA plays a pivotal role in binding host cell receptors and releasing the viral RNA into the cell (Beer et al. 2018). It contains a receptor-binding site (RBS) in a globular head domain which is composed of three domains, the 130-loop (residues 134-138), the 190-helix (residues 188-195), and the 220-loop (residues 221-228), and targets sialic acid residues on host cells (Skehel and Wiley 2000; Mair et al. 2014; Tzarum et al. 2015). Four main canonical epitopes, $\mathrm{Ca}$ (including $\mathrm{Ca} 1$ and $\mathrm{Ca} 2), \mathrm{Cb}$, $\mathrm{Sa}$, and $\mathrm{Sb}$, on the globular HA head have been recognized by the host immune system. To prevent them from being blocked by antibodies elicited due to vaccination and natural infection, changes in HA protein associated with antigenic drift happen continually over time as the virus replicates (Caton et al. 1982; Martinez et al. 2009). Circulating influenza viruses evade neutralization in their human hosts by acquiring escape mutations. Nevertheless, it might result in the change of structure and biological functions of HA and makes mutant virus easier for reinfecting humans if escape mutations residues overlap the RBS region.

HA K166Q (H3 numbering without signal peptide), is believed to be the first antigenic drift site identified by an epitope mapping approach and became dominant in the 2013-2014 influenza season (Yasuhara et al. 2017). Christopher et al. identified seven distinct amino acid substitutions in the HA segment under the selective pressure of a human monoclonal antibody (EM4C04), six of which significantly reduced binding activity to EM4C04
(K123N, D131E, K133T, G134S, K157N, and G158E) and one of which, S186P, increased the binding affinity of HA to the receptor (O'Donnell et al. 2012). According to the GISAID Flu database, S186P did not become dominant until 2018.

An escape mutation $\mathrm{N} 159 \mathrm{~K}$ was identified as being responsible for the antigenic drift in the epitope $\mathrm{Sa}$ in the ferret (Guarnaccia et al. 2013). Rudneva et al. reported five amino acid substitutions in the epitopes Sa (N129S, K156E, G158E, and N159D) and Sb (D190N) (Rudneva et al. 2012). Chen et al. showed A144E and K145E substitutions in the epitope $\mathrm{Ca} 2, \mathrm{G} 158 \mathrm{E}$ in the epitope $\mathrm{Sa}$, G173E in the epitope Ca1, and S188N and Q192E mutations in the epitope $\mathrm{Sb}$ by the epitope mapping strategy (Chen et al. 2014).

Here we report an influenza $\mathrm{A}(\mathrm{H} 1 \mathrm{~N} 1)$ pdm09-infected clinical isolate which contained four substitutions in the epitope Sb (S186P, T188I, D190A, and Q192E). We analyzed the evolution of this antigenic drift motif, characterized its pathogenicity, and evaluated its transmissibility based on the vaccine strains recommended by WHO in the northern and southern hemisphere in the 2020-2021 influenza season.

\section{Materials and Methods}

\section{Case}

On December 14, 2019, a 46-year-old male patient was enrolled in the Guangzhou No.8 People's Hospital (Guangdong, China) with fever $\left(38.8^{\circ} \mathrm{C}\right)$ and respiratory symptoms. Bronchoalveolar lavage fluid was analyzed in a broad spectrum of microorganisms by metagenomic nextgeneration sequencing (mNGS) and confirmed to be positive for influenza A (H1N1) pdm09 virus.

\section{Virus Isolation}

Two clinical isolates, A/Guangdong/GLW/2018 (GLW/18) and $\mathrm{A} /$ Guangdong/LCF/2019 (GLW/19), were used in this study. The former viral RNA was extracted from patients' swab and the latter one from bronchoalveolar lavage fluid, respectively. Both cDNAs were generated by SuperScript $^{\mathrm{TM}}$ III Reverse Transcriptase (Invitrogen) (Eisfeld et al. 2014). Full genome sequences of both strains were determined by the Sanger Sequencing Method and assembled by the Vector NTI suite. Genome sequences were deposited in the GISAID's EpiFlu ${ }^{\mathrm{TM}}$ Database: A/Guangdong/GLW/2018, EPI_ISL_335963; A/Guangdong/LCF/2019, EPI_ISL_412027. 


\section{Generation of Recombinant Viruses}

Two strains, GLW/18 and LCF/19 were selected as the backbone for constructing different reassortant viruses to characterize and compare the 186-192 motif's pathogenicity on the HA segment. According to the manufacturer's instructions, viral RNAs of both isolates were extracted using a QIAamp Viral RNA Mini Kit (Qiagen, Valencia, CA). Full-length viral cDNA segments were amplified using reverse transcription-PCR with segmentspecific primers as described (Hoffmann et al. 2001), and cloned into the pHW2000-derived vector using a ligationindependent cloning method (protocol is available upon request). GLW/18-S186P and GLW/18-S186P-T188I mutagenesis plasmids were constructed using the QuikChange II site-directed mutagenesis kit (Agilent). Genotypes of reassortant viruses used in this study are listed in Table 1.

Plasmids were sequenced to confirm that no mutations were introduced during the cloning procedure. Recombinant viruses and their parental prototype viruses were generated by transfecting eight plasmids into mixed MDCK and HEK293T cells, as described previously (Hoffmann et al. 2000), using a TransIT LT-1 kit (Mirus, Madison, WI). All influenza viruses used in this study were rescued using the reverse genetics (RG) approach. RG viruses were sequence-confirmed and propagated in MDCK cells at $37{ }^{\circ} \mathrm{C}$ for two days (P1) using the standard protocol (Song et al. 2014).

\section{Growth Kinetics}

Viral titration was used for the determination of their growth curves. A standard growth kinetics approach was applied in this study. Briefly, MDCK cells were inoculated with each RG virus at $\mathrm{MOI}=0.001$ (multiplicity of infection) and incubated at $37{ }^{\circ} \mathrm{C}$ supplied with $5 \% \mathrm{CO}_{2}$ in the minimal essential medium (MEM, GIBCO) containing TPCK-treated trypsin (GIBCO). Virus culture media were harvested at $12,24,48$, and $72 \mathrm{~h}$ post-infection (h.p.i.), and the viruses were titrated in MDCK cells by plaque assay. The viral growth property was measured in MDCK cells with three independent wells as previously described (Wang et al. 2019).

\section{Plaque Assay}

Viruses were serially diluted tenfold and absorbed onto confluent MDCK cells in 6-well plates and then incubated at $37{ }^{\circ} \mathrm{C}$ for $1 \mathrm{~h}$. The supernatant was removed, and the cells were washed with PBS and then overlaid with $1 \%$ MEM agarose containing $2 \mu \mathrm{g} / \mathrm{mL}$ L-(tosylamide-2-phenyl) ethyl chloromethyl ketone (TPCK)-treated trypsin. The plates were incubated at $37{ }^{\circ} \mathrm{C}$ for $48 \mathrm{~h}$ and then fixed with $4 \%$ formaldehyde in $1 \times$ PBS buffer for at least $2 \mathrm{~h}$. Plaques were visualized by staining with $1 \%$ crystal violet in $20 \%$ alcohol solution.

\section{Hemagglutination Inhibition (HI) Assay}

HI tests with recombinant viruses were conducted as described (Wu et al. 2008). Briefly, recovered patient sera collected from the GLW/18-infected patient were treated with a receptor-destroying enzyme (RDE, Denka Seiken Co., Tokyo, Japan). Control antibodies and sera were then twofold serially diluted in 96-well plates. Subsequently, 8 HA units of the virus were added to each well and incubated at room temperature for $1 \mathrm{~h}$. Then, $50 \mu \mathrm{L}$ of $0.5 \%$ Turkey erythrocytes (Lampire Biological Laboratories, PA) was added to the serum/virus mixture followed by incubation for $30 \mathrm{~min}$. The HI titer is defined as the reciprocal of the highest dilution of sera that completely
Table 1 Genotypes and HI titers of the GLW/18, LCF/19 recombinant viruses and reassorted mutants.

\begin{tabular}{|c|c|c|c|c|c|c|c|c|c|}
\hline \multirow[t]{3}{*}{ H3 numbering } & \multicolumn{7}{|l|}{ HA1 } & \multirow{3}{*}{$\begin{array}{l}\text { HA2 } \\
\frac{154}{}\end{array}$} & \multirow[b]{3}{*}{$\mathrm{HI}$} \\
\hline & \multicolumn{7}{|c|}{ Epitope $\mathrm{Sb}$ region } & & \\
\hline & 186 & 188 & 190 & 192 & 120 & 129 & 260 & & \\
\hline A/California/4/2009* & & & & & & & & & $>20,480$ \\
\hline GLW/18 & $\mathrm{S}$ & $\mathrm{T}$ & $\mathrm{D}$ & Q & A & $\mathrm{N}$ & $\mathrm{N}$ & $\mathrm{R}$ & 2560 \\
\hline $\mathrm{LCF} / 19$ & $\mathrm{P}$ & I & A & $\mathrm{E}$ & $\mathrm{T}$ & $\mathrm{D}$ & $\mathrm{D}$ & G & 320 \\
\hline GLW/18-LCF-HA & $\mathrm{P}$ & I & A & $\mathrm{E}$ & $\mathrm{T}$ & $\mathrm{D}$ & $\mathrm{D}$ & G & 160 \\
\hline GLW/18-LCF-HA-chimera & $\mathrm{P}$ & I & A & $\mathrm{E}$ & A & $\mathrm{N}$ & $\mathrm{N}$ & $\mathrm{R}$ & 320 \\
\hline LCF/19-GLW-HA & $\mathrm{S}$ & $\mathrm{T}$ & $\mathrm{D}$ & Q & A & $\mathrm{N}$ & $\mathrm{N}$ & $\mathrm{R}$ & 2560 \\
\hline LCF/19-GLW-HA-chimera & $\mathrm{S}$ & $\mathrm{T}$ & $\mathrm{D}$ & Q & $\mathrm{T}$ & $\mathrm{D}$ & $\mathrm{D}$ & G & 2560 \\
\hline GLW/18-HA-S186P & $\mathrm{P}$ & $\mathrm{T}$ & $\mathrm{D}$ & Q & A & $\mathrm{N}$ & $\mathrm{N}$ & $\mathrm{R}$ & 640 \\
\hline GLW/18-HA-S186P-T188I & $\mathrm{P}$ & I & $\mathrm{D}$ & Q & A & $\mathrm{N}$ & $\mathrm{N}$ & $\mathrm{R}$ & 160 \\
\hline
\end{tabular}

*A/California/4/2009 RG version is used as a standard strain. 
inhibits hemagglutination. The HI test was started at a 1:20 dilution for anti-GLW/18 serum.

\section{Bioinformatics}

HA segment sequences of pdm09 viruses were downloaded from the GISAID flu database. The phylogenetic tree was constructed using MEGA 7.0 by the Neighbor-Joining (NJ) method with 1000 bootstrap replicates.

\section{Statistical Analysis}

GraphPad Prism ver. 8.02 for Windows (GraphPad Software, USA) was used to analyze the data. Two-way ANOVA was performed. Probability values of less than $0.01(P<0.05)$ were considered statistically significant. No samples were excluded from the analysis.

\section{Results}

\section{Clinical Specimen}

The patient was a 46-year-old man with hypertension, diabetes, chronic heart failure, viral hepatitis (type B), and gallbladder polyps who lived in Guangdong Province. Radiograph imaging of the chest was recorded during the disease progression (Fig. 1).

From December 14 to 23, 2019, the patient was admitted to the Guangzhou No.8 People's Hospital. On day 1, the patient started coughing, had a fever $\left(38.8^{\circ} \mathrm{C}\right)$, dizziness, chills, and fatigue symptoms. On the same day, shortness of breath developed, and blood oxygen saturation was approximately $91 \%$. The patient was diagnosed with severe pneumonia, acute respiratory distress syndrome (ARDS), and type II respiratory failure. On day 2, H1N1 was detected in his bronchoalveolar lavage fluid; therefore, the patient was treated with Tamiflu (oseltamivir phosphate, $150 \mathrm{mg} / \mathrm{d}$ ). On day 3, a chest X-ray showed multiple large pieces of shadows in bilateral lungs and partial blurring on the right diaphragm. On day 10, December 24, the patient was transferred to the First Affiliated Hospital of Guangzhou Medical University. The patient's upper respiratory tract symptom onset accompanied by fever rapidly progressed to respiratory failure and multi-visceral failure. The pandemic H1N1 nucleic acid of influenza A virus was still detected as positive, and the patient was treated with meropenem, voriconazole, and oseltamivir. On day 32, the patient had no symptoms such as cough, shortness of breath, dizziness, chills, fatigue, and body temperature $\left(37.5^{\circ} \mathrm{C}\right)$. Influenza A H1N1 virus test was negative. On day 39 , the patient's condition was stable, and he was transferred from the intensive care department to the general ward. On day 63, the patient was recovered and was discharged (February 15, 2020). However, serum antibodies against anti-GLW/18 were not at a high concentration in vivo $(\mathrm{HI}=40)$, suggesting that 2019 pdm09 H1N1 might undergo antigenic change.

\section{Molecular Evolution of the 186-192 motif of the pdm09 HA Segment}

We analyzed 41,987 full-length HA segment sequences of influenza A H1N1 pdm09 viruses downloaded from the GISAID database (cut-off date: August 24, 2020), 40708, which correspond to $97 \%$ of the sequences, were mainly classified into five genotypes based on the 186-192 motif. The earliest HA genotype containing a CA4-like motif (STSADQQ) was gradually replaced by a GLW-like motif (STTADQQ). The appearance of HA S188T and S186P in the epitope $\mathrm{Sb}$ also occurred in the coming year of the 2009 swine flu outbreak, although the substitutions became dominant in 2016 and 2019, respectively. A threonine-toisoleucine substitution at HA 188 emerged in 2017, and its isolation rate reached a higher level in 2019 and the isolate with the PTIAAQE motif is circulating presently (Fig. 2A). The outbreak of the coronavirus SARS-CoV-2 led to reduced influenza surveillance and reporting activities in
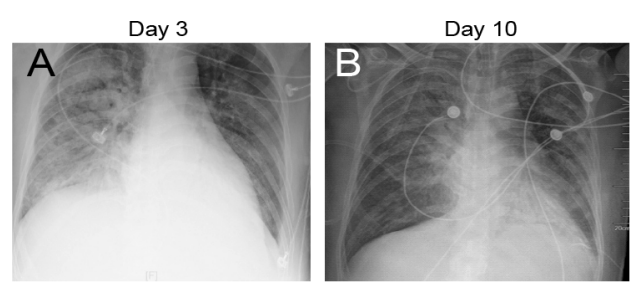

Fig. 1 Representative radiographic findings of influenza A (H1N1) pdm09 virus. Clinical course of a 46-year-old man with hypertension, diabetes, chronic heart failure, viral hepatitis (type B), and hypoproteinemia infected with influenza A (H1N1) pdm09 virus, Guangdong, China, in 2019. A Radiograph imaging of the chest taken showed large and patchy shadows of both lungs with blurred edges, mainly the right lung, and partial blurring on the right diaphragm on the 3rd

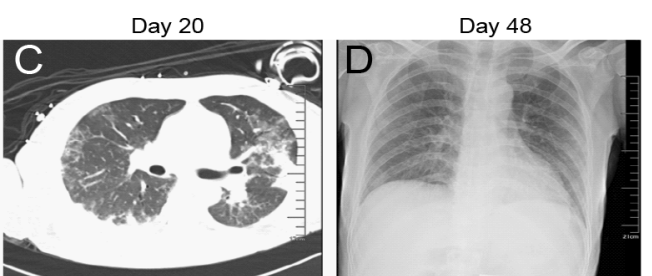

day after hospitalization; B Image taken on the 10th day, showing improvement of both lungs; C computed tomographic (CT) scan of the chest showed bilateral ground-glass opacities at the periphery of both lungs at day 20; D Image taken in the convalescent period of the infection on day 48 , the X-ray film showed that the lung lesions were basically absorbed. 
A

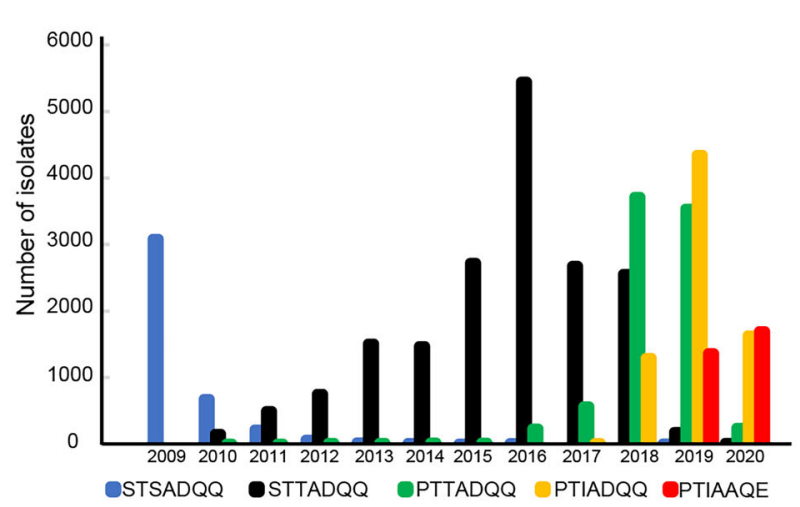

B

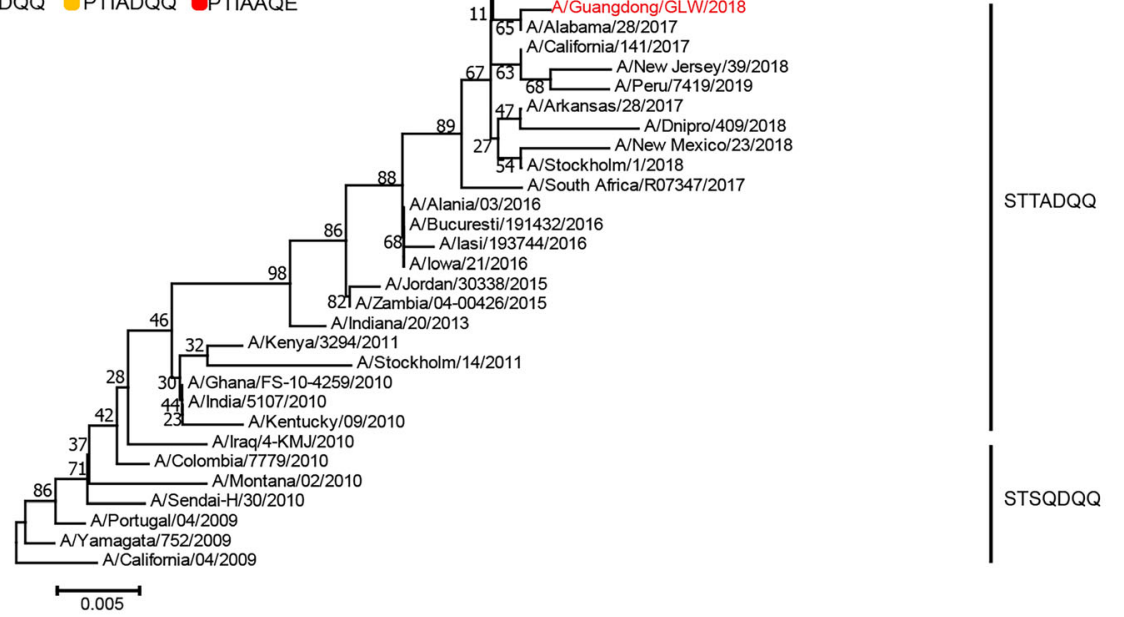

Fig. 2 Evolution of hemagglutinin protein of influenza A(H1N1)pdm09 virus. A Distribution of the hemagglutinin 186-192 motif; B The phylogenetic tree of hemagglutinin protein

many countries. Additionally, travel restrictions and socialdistancing measures in several countries resulted in decreased influenza activity. Overall, fewer viruses were available for characterization after August 2020.

Interestingly, as a part of the epitope $\mathrm{Sb}$, the 190 helix appeared in two amino acid substitutions D190A and Q192E in July 2019 and quickly became dominant in the pdm09 isolates, suggesting that such isolates (LCF-like motif PTIAAQE) adapted better to the population than the isolates containing the PTIADQQ motif and the PTTADQQ motif (Fig. 2B).

\section{HA S186P Enhances H1N1 Virus Propagation in Madin-Darby Canine Kidney (MDCK) Cells}

To examine the effect of viral replication, eight reverse genetics (RG) versions of pdm09 recombinant viruses (shown in Table 1) were infected in MDCK cells at an MOI of 0.001. We first compared the growth curves of two wild types, and our results showed that $\mathrm{LCF} / 19$ replicated faster than GLW/18 at 12, 24, and 48 h.p.i. $(P<0.01$ at 24 h.p.i. of influenza $\mathrm{A}(\mathrm{H} 1 \mathrm{~N} 1) \mathrm{pdm} 09$ virus. The phylogenetic trees were generated using MEGA 7.0 by the Neighbor-Joining (NJ) method.

and $P<0.05$ at 48 h.p.i.) (Fig. 3A). To exclude factors from other segments, GLW/18-LCF-HA and LCF/19GLW-HA recombinant viruses were generated.

The mean value obtained from triplicates showed that the LCF-HA segment grew faster than the latter with GLW-HA (no statistically significant difference) (Fig. 3A). Furthermore, to confirm that the 186-192 substitutive motif increased virus replication, RG versions of GLW/18-LCF and GLW/18-LCF HA chimeric segments were rescued. Both RG viruses grew faster than GLW/18 WT $(P<0.05$ at 24 and 48 h.p.i.) (Fig. 3A). Overall, RG recombinant viruses containing the four substitutions on the epitope $\mathrm{Sb}$ replicated faster than those with GLW/18 HA genotypes in MDCK cells. Combined with the clinical data, mutagenesis that happened on the epitope $\mathrm{Sb}$ of the HA segment might increase the pdm09 viral pathogenicity in vivo.

To examine natural isolates containing the PTTADQQ motif and the PTIADQQ motif, two RG viruses, GLW/18HA-S186P and GLW/18-HA-S186P-T188I, were generated to determine their growth kinetics curves. The GLW/ 18-derived recombinant viruses' growth curves were 

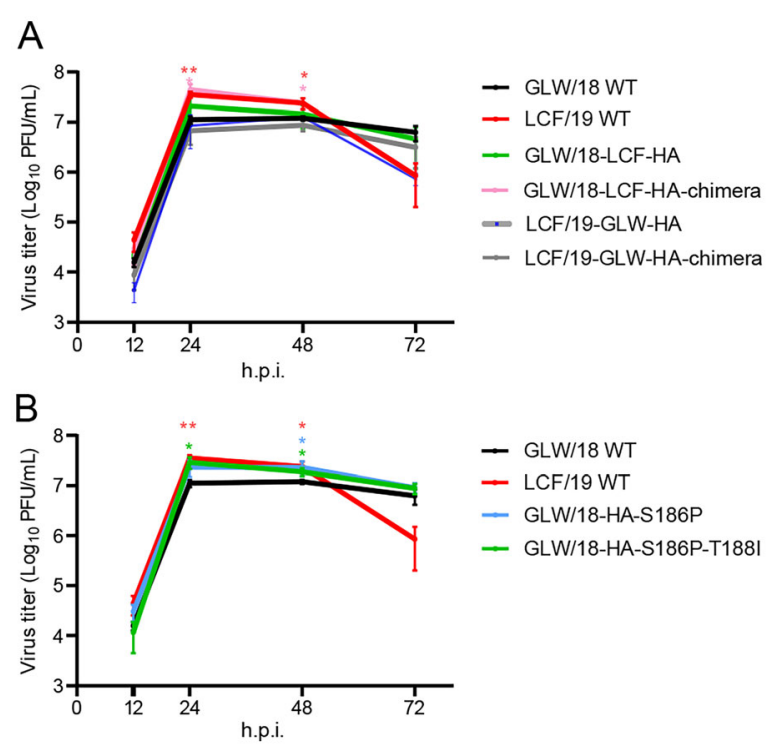

Fig. 3 Growth curves of the GLW/18, LCF/19 recombinant viruses and reassorted mutants. The growth kinetics of the wild-type GLW/18 RG virus and the indicated mutant viruses in MDCK cells were compared. Cell culture supernatants of MDCK cells infected at an MOI of 0.001 were collected at 12, 24, 48, and 72 h.p.i.. Virus titers are presented as the mean $\pm \mathrm{SD}(\mathrm{n}=3)$. $* * P<0.01, * P<0.05$ (one-way ANOVA).

similar. Both demonstrated higher propagation velocities than GLW/18 WT at 24 and 48 h.p.i., respectively, indicating that the proline residue at HA 186 is a critical amino acid to decide the virus propagation $(P<0.05)$ (Fig. 3B).

\section{The Escape Mutation in the Epitope Sb Decreased the Binding Activity Against GLW/18 Serum}

To understand the molecular basis of the antigenic variation of pdm09 viruses since 2017, an HI test was performed (Table 1). Serum collected from GLW/18-infected patients was subjected to an HI test against GLW/18, whose HI titer was 2560. RG viruses containing the 186-192 motif STTADQQ of HA segments in the epitope Sb demonstrated at least eight times higher than those having the PTIAAQE. Of these mutations, HA S186P and T188I are critical in changing the binding activity to the sera. It seems that D190A and Q192E did not affect HI titers significantly.

\section{The Vaccine Recommended for the Southern Hemisphere does not Contain the PTIAAQE Mutation}

The quadrivalent or trivalent influenza vaccine strains recommended by WHO in the 2020-2021 northern hemisphere influenza season were A/Guangdong-Maonan/ SWL1536/2019 A/Hawaii/70/2019, both of which contain
PTIAAQE mutation motif; these vaccines could prevent people from infection and severe outcomes caused by influenza viruses. (https://www.who.int/influenza/vaccines/ virus/recommendations/en/). However, the vaccine strains recommended in the southern hemisphere between 2020 and 2021 do not include such mutation strains (A/Brisbane/ 02/2018 for use in 2020; A/Victoria/2570/2019 and A/Wisconsin/588/2019 for use in 2021) (Table 2). The GISAID database showed a few cases infected with pdm09 viruses carrying the HA segment's PTIAAQE mutation. Due to the difference in the selection of vaccine strains, people vaccinated in the southern hemisphere could still suffer a serious infection if infected with this antigenic drift-resulting pdm09 virus.

Taken together, a novel antigenic drift in the epitope $\mathrm{Sb}$ of the pdm09 virus was revealed by bioinformatic analysis and serological study. Currently, pdm09 viruses circulating in the northern hemisphere containing this mutated motif adapted well to human populations and are becoming dominant strains in the coming influenza season. Vaccine strains recommended for use in the northern hemisphere could protect against this escape mutant well. However, they may offer only partial protection against influenza infection if it quickly becomes a dominant strain in the southern hemisphere in winter 2020.

\section{Discussion}

Influenza $\mathrm{A}$ virus is a serious global health threat that imposes an enormous economic and health burden, potentially causing periodic catastrophic pandemics (Kosik and Yewdell 2019). Among humans, the annual epidemic influenza illness is caused by two types, influenza $\mathrm{A}(\mathrm{H} 1 \mathrm{~N} 1)$ and influenza $\mathrm{A}(\mathrm{H} 3 \mathrm{~N} 2)$, which have been common influenza viral subtypes circulating among humans since the late 1970s (Cox and Subbarao 2000). In April 2009, a swine-origin $\mathrm{A}(\mathrm{H} 1 \mathrm{~N} 1)$ virus (pdm09), taking over the previously circulating seasonal $\mathrm{A}(\mathrm{H} 1 \mathrm{~N} 1)$ virus, caused the first influenza A virus pandemic of the twenty-first century (Novel Swine-Origin Influenza AVIT et al. 2009), and continues to cocirculate with the seasonal $\mathrm{A}(\mathrm{H} 3 \mathrm{~N} 2)$ virus. Influenza $\mathrm{A}(\mathrm{H} 3 \mathrm{~N} 2)$ appeared in the late 1960s and circulated among humans for over 50 years; therefore, two surface glycoproteins, hemagglutinin and neuraminidase, were evolved various mutations to evade the host immune system. Unlike influenza A(H3N2), the history of influenza A (H1N1) pdm09 is much shorter than that of influenza $\mathrm{A}(\mathrm{H} 3 \mathrm{~N} 2)$ circulating in the human population. Therefore, its surface proteins keep more conserved regions compared to its earliest isolate.

The two H1N1 pdm09 strains used in this study were GLW/18, isolated from a 7-year-old boy, and LCF/19, 
Table 2 Genotypes of vaccine strains recommended by WHO in the southern and northern hemispheres in 2020-2021.

\begin{tabular}{|c|c|c|c|c|c|c|c|}
\hline & \multirow[t]{2}{*}{ Year } & \multirow[t]{2}{*}{ Vaccine type } & \multirow[t]{2}{*}{ Strain name } & \multicolumn{4}{|c|}{$\mathrm{HA}$ Epitope $\mathrm{Sb}$ region } \\
\hline & & & & 186 & 188 & 190 & 192 \\
\hline \multirow[t]{2}{*}{ Northern hemisphere } & $2020-2021$ & Egg-, cell- or recombinant-based & A/Guangdong-Maonan/SWL1536/2019 & $\mathrm{P}$ & I & A & $\mathrm{E}$ \\
\hline & & & A/Hawaii/70/2019 & $\mathrm{P}$ & I & A & $\mathrm{E}$ \\
\hline \multirow[t]{3}{*}{ Southern hemisphere } & 2021 & Cell- or recombinant- based & A/Wisconsin/588/2019 & $\mathrm{P}$ & I & $\mathrm{D}$ & Q \\
\hline & 2021 & Egg-based & A/Victoria/2570/2019 & $\mathrm{P}$ & I & $\mathrm{D}$ & Q \\
\hline & 2020 & & A/Brisbane/02/2018 & $\mathrm{P}$ & $\mathrm{T}$ & $\mathrm{D}$ & Q \\
\hline
\end{tabular}

collected from a 46-year-old man with many diseases. Children, the elderly, and people with weakened immune systems are susceptible to being infected by the influenza A (H1N1) pdm09 virus.

To our knowledge, the first epitope mutation (HA K166Q) was identified in the clinical isolates in 2017 that showed superior growth to that of the wild-type virus (Yasuhara et al. 2017). Both GLW/18 and LCF/19 clinical isolates also carry this escape mutation, suggesting that the HA K166Q is dominant in the epidemic strains. H1N1 pdm09 isolates containing HA S186P and T188I appeared in 2010 and 2017, respectively, but gradually became dominant in 2018. Moreover, double substitutions D190A and Q192E on the 190-helix domain occurred in Mid-2019, and its isolation is high, suggesting that those two residues, A190 and E192, at the 190-helix might have a much more stable structure than D190 and Q192. Therefore, the virus containing the escape mutant PTIAAQE (186-192) in the epitope $\mathrm{Sb}$ has become a stable strain in the population, indicating that it is easily circulating in the coming winter seasonal flu epidemics the northern hemisphere. A190V egg-adapted substitution occurred in WHO recommended vaccines, A/Guangdong-Maonan/SWL1536/2019 and A/Hawaii/70/2019, after serial passages in eggs. It is unclear whether this substitution will become dominant soon. The accumulated antigenic drift that finally causes antigenic shift might represent a pressing public health challenge.

Due to the coronavirus (COVID-19) pandemic, few flu data were uploaded to the GISAID Flu database after August 2020. We are concerned that the people living in the southern hemisphere could not obtain full protection if immunized with a vaccine lacking this mutation.

COVID-19 continues its spread globally and has now passed 50 million confirmed cases in 190 countries. Both viruses have similar transmission characteristics and common clinical manifestations but different treatments. Coinfection cases have been reported in different research groups (Konala et al. 2020; Ozaras et al. 2020; Singh et al. 2020). It is unclear whether patients infected have a worse prognosis than those in whom SARS-CoV-2 is the only detected pathogen. Since vaccine additives can enhance immune flexibility, flu vaccine immunized-people might produce immune responses against COVID-19 if infected with SARS-CoV-2.

Acknowledgements This work was supported by the National Natural Science Foundation of China (Grant No. 82074311), the General Project of Guangzhou Medical University (Grant No.SKLRD-MS201908), and the Yunnan Provincial Science and Technology Department (Grant No. 202005AF150043). We would like to thank Robert Webster of St Jude Children Research Hospital, USA, for providing the pHW2000 vector; Dr. Kai Huang and Dr. Pui Wang for constructive comments.

Author Contributions LX and YC performed the research and analyzed the results. BC, LB, YL, ZZ, WG, QC, and YL performed virus isolation, analyzed the clinical data. HC, XD and XW co-directed the study. KQ and WS directed the study, analyzed, interpreted results and wrote the manuscript.

\section{Compliance with Ethical Standards}

Conflict of interest The authors declare no conflict of interest.

Animal and Human Rights Statement The studies involving human participants were reviewed and approved by the Ethics Committee of The First Affiliated Hospital of Guangzhou Medical University (File No. 2014-50 approved on June 2, 2015). The patients/participants provided their written informed consent to participate in this study.

\section{References}

Beer K, Dai M, Howell S, Rijal P, Townsend AR, Lin Y, Wharton SA, Daniels RS, McCauley JW (2018) Characterization of neutralizing epitopes in antigenic site $\mathrm{B}$ of recently circulating influenza A(H3N2) viruses. J Gen Virol 99:1001-1011

Caton AJ, Brownlee GG, Yewdell JW, Gerhard W (1982) The antigenic structure of the influenza virus $\mathrm{A} / \mathrm{PR} / 8 / 34$ hemagglutinin (H1 subtype). Cell 31:417-427

Chen J, Yan B, Chen Q, Yao Y, Wang H, Liu Q, Zhang S, Wang H, Chen Z (2014) Evaluation of neutralizing efficacy of monoclonal antibodies specific for 2009 pandemic H1N1 influenza A virus in vitro and in vivo. Arch Virol 159:471-483

Cox NJ, Subbarao K (2000) Global epidemiology of influenza: past and present. Annu Rev Med 51:407-421 
Eisfeld AJ, Neumann G, Kawaoka Y (2014) Influenza A virus isolation, culture and identification. Nat Protoc 9:2663-2681

Guarnaccia T, Carolan LA, Maurer-Stroh S, Lee RT, Job E, Reading PC, Petrie S, McCaw JM, McVernon J, Hurt AC, Kelso A, Mosse J, Barr IG, Laurie KL (2013) Antigenic drift of the pandemic $2009 \mathrm{~A}(\mathrm{H} 1 \mathrm{~N} 1)$ influenza virus in A ferret model. PLoS Pathog 9:1003354

Hoffmann E, Neumann G, Kawaoka Y, Hobom G, Webster RG (2000) A DNA transfection system for generation of influenza A virus from eight plasmids. Proc Natl Acad Sci U S A 97:6108-6113

Hoffmann E, Stech J, Guan Y, Webster RG, Perez DR (2001) Universal primer set for the full-length amplification of all influenza A viruses. Arch Virol 146:2275-2289

Konala VM, Adapa S, Gayam V, Naramala S, Daggubati SR, Kammari CB, Chenna A (2020) Co-infection with Influenza A and COVID-19. Eur J Case Rep Intern Med 7:001656

Kosik I, Yewdell JW (2019) Influenza hemagglutinin and neuraminidase: Yin(-)Yang proteins coevolving to Thwart immunity. Viruses 11:346

Latorre-Margalef N, Tolf C, Grosbois V, Avril A, Bengtsson D, Wille M, Osterhaus AD, Fouchier RA, Olsen B, Waldenstrom J (2014) Long-term variation in influenza A virus prevalence and subtype diversity in migratory mallards in northern Europe. Proc Biol Sci 281:20140098

Mair CM, Ludwig K, Herrmann A, Sieben C (2014) Receptor binding and $\mathrm{pH}$ stability: how influenza $\mathrm{A}$ virus hemagglutinin affects host-specific virus infection. Biochim Biophys Acta 1838:1153-1168

Martinez O, Tsibane T, Basler CF (2009) Neutralizing anti-influenza virus monoclonal antibodies: therapeutics and tools for discovery. Int Rev Immunol 28:69-92

Novel Swine-Origin Influenza AVIT, Dawood FS, Jain S, Finelli L, Shaw MW, Lindstrom S, Garten RJ, Gubareva LV, Xu X, Bridges CB, Uyeki TM (2009) Emergence of a novel swineorigin influenza A (H1N1) virus in humans. N Engl J Med 360:2605-2615

O'Donnell CD, Vogel L, Wright A, Das SR, Wrammert J, Li GM, McCausland M, Zheng NY, Yewdell JW, Ahmed R, Wilson PC, Subbarao K (2012) Antibody pressure by a human monoclonal antibody targeting the 2009 pandemic H1N1 virus hemagglutinin drives the emergence of a virus with increased virulence in mice. mBio 3:e00120-12

Ozaras R, Cirpin R, Duran A, Duman H, Arslan O, Bakcan Y, Kaya M, Mutlu H, Isayeva L, Kebanli F, Deger BA, Bekeshev E, Kaya F, Bilir S (2020) Influenza and COVID-19 coinfection: report of six cases and review of the literature. J Med Virol 92:2657-2665

Rudneva I, Ignatieva A, Timofeeva T, Shilov A, Kushch A, Masalova O, Klimova R, Bovin N, Mochalova L, Kaverin N (2012) Escape mutants of pandemic influenza A/H1N1 2009 virus: variations in antigenic specificity and receptor affinity of the hemagglutinin. Virus Res 166:61-67

Singh B, Kaur P, Reid RJ, Shamoon F, Bikkina M (2020) COVID-19 and influenza co-infection: report of three cases. Cureus 12:e9852

Skehel JJ, Wiley DC (2000) Receptor binding and membrane fusion in virus entry: the influenza hemagglutinin. Annu Rev Biochem 69:531-569

Song W, Wang P, Mok BW, Lau SY, Huang X, Wu WL, Zheng M, Wen X, Yang S, Chen Y, Li L, Yuen KY, Chen H (2014) The K526R substitution in viral protein PB2 enhances the effects of E627K on influenza virus replication. Nat Commun 5:5509

Tzarum N, de Vries RP, Zhu X, Yu W, McBride R, Paulson JC, Wilson IA (2015) Structure and receptor binding of the hemagglutinin from a human H6N1 influenza virus. Cell Host Microbe 17:369-376

Wang P, Song W, Mok BW, Zheng M, Lau SY, Liu S, Chen P, Huang X, Liu H, Cremin CJ, Chen H (2019) The PB2 polymerase host adaptation substitutions prime Avian Indonesia Sub Clade 2.1 H5N1 Viruses for infecting humans. Viruses 11:292

Wu WL, Chen Y, Wang P, Song W, Lau SY, Rayner JM, Smith GJ, Webster RG, Peiris JS, Lin T, Xia N, Guan Y, Chen H (2008) Antigenic profile of avian H5N1 viruses in Asia from 2002 to 2007. J Virol 82:1798-1807

Wu Y, Wu Y, Tefsen B, Shi Y, Gao GF (2014) Bat-derived influenzalike viruses H17N10 and H18N11. Trends Microbiol 22:183-191

Yasuhara A, Yamayoshi S, Soni P, Takenaga T, Kawakami C, Takashita E, Sakai-Tagawa Y, Uraki R, Ito M, IwatsukiHorimoto K, Sasaki T, Ikuta K, Yamada S, Kawaoka Y (2017) Diversity of antigenic mutants of influenza A(H1N1)pdm09 virus escaped from human monoclonal antibodies. Sci Rep $7: 17735$ 